\section{British Society for Paediatric Dermatology}

\section{IMPACT OF DIVERSITY IN TRAINING RESOURCES ON SELF-CONFIDENCE IN DIAGNOSING SKIN CONDITIONS ACROSS A RANGE OF SKIN TONES: AN INTERNATIONAL SURVEY}

${ }^{1}$ Shaarna Shanmugavadivel, ${ }^{2}$ Danilo Buonsenso, ${ }^{1}$ Jo-Fen Liu, ${ }^{3}$ Tessa Davis, ${ }^{4}$ Damian Roland. ${ }^{1}$ University of Nottingham; ${ }^{2}$ Department of Women and Child Health and Public Health, Fondazione Policlinico Universitario A. Gemelli IRCCS, Rome, Italy; ${ }^{3}$ Paediatric Emergency Department, Royal London Hospital, London UK; ${ }^{4}$ Paediatric Emergency Medicine Leicester Academic (PEMLA) Group, Children's Emergency Department, Leicester Royal Infirmary, Leicester, UK

\subsection{6/archdischild-2021-rcpch.288}

Background There is increasing recognition of the lack of diversity representation in all areas of medicine, in particular within medical education. Studies assessing diversity of skin tone image representation among textbooks and major scientific journals showed that the majority of images reported are in children with light skin tones. However, the impact of the lack of diversity of skin tone representation in medical resources on the ability of healthcare workers to recognise childhood skin conditions has not been explored.

Objectives To assess diversity in medical training resources and its impact on self-confidence in diagnosing skin conditions on different skin tones of healthcare workers across the globe.

Methods Participants were asked to $\log$ in to the online platform (www.dftbskindeep.com) and self-declare the following information: continent of practice, ethnicity, profession, specialty, years of experience, majority Training Resources (white skin, a mix of skin tones, darker skin tones only), and confidence in diagnosing skin conditions. Data analyses were performed using IBM SPSS 26.0 for Windows (IBM Corp, Armonk, NY USA). Categorical variables were presented as proportions, and Chi-squared or Fisher's exact tests were used to compare the distribution between groups as appropriate. A p-value of $<0.05$ was deemed statistically significant in all analyses.

Results 600 eligible participants were included in the analysis.

\section{Demographics}

Participants responded from all continents, although Europe $(56 \%)$ and Oceania (23\%) were the most represented. The majority of participants were white/Caucasian (69\%). 439 $(73 \%)$ were clinicians, mostly paediatricians (37\%), emergency doctors (22\%) paediatric emergency doctors (12\%) and a minority were dermatologists (3\%). Just over half of participants $(56 \%)$ reported at least six years of practice since graduation.

\section{Training resources}

Participants were asked to report the majority of training resources used during their training. 441 (74\%) reported that only white skin was usually represented, while resources including a mix of skin tones $(24 \%)$ or other skin tones $(3 \%)$ were rarely used.

\section{Self-confidence}

When diagnosing skin conditions in darker skin tones, participants reported that they are 'generally uncertain if correct' in $43 \%$ cases, 'sometimes uncertain but clinically safe' (43\%), and 'confident across a range of skin tones' in a minority of cases $(5 \%)$.

Self-confidence levels were associated with geographical location (higher in Africa (4/14, 29\%) and Latin America (1/9,
11\%), $(\mathrm{P}<0.001)$; diversity of training resources used (higher with a mix of skin tones $(15 / 144,10 \%)$ or darker skin tones only $(3 / 15,20 \%)(<0.001)$; with longer working experience (6 to $10(9 / 188,5 \%)$ or $>10$ years of practice $(18 / 159,11 \%)(\mathrm{P}<0.001)$ and with specialty (higher in dermatologists $(9 / 17,53 \%, \mathrm{P}<0.001)$. Self-confidence was very low among paediatricians, emergency medicine and paediatric emergency medicine specialists $(<5 \%)$. Ethnicity did not seem to be associated with self-confidence $(\mathrm{P}>0.05)$.

Conclusions Our study provides further support of the lack of diversity in training resources used by healthcare professionals on a global scale. Moreover, our findings provide preliminary evidence that this lack of diversity affects self-confidence in diagnosing skin conditions on a variety of skin tones.

\section{British Association of Perinatal Medicine and Neonatal Society}

\section{MISSED AND DELAYED NEWBORN BLOOD SPOT SCREENING IN SPECIALIST HOSPITALS AND NEONATAL UNITS}

Helen Lewis-Parmar, Michele Seddon, Christine Cavanagh. Public Health UK

\subsection{6/archdischild-2021-rcpch.289}

Background Delayed diagnosis means delayed initiation of treatment for screen positive babies in the newborn blood spot screening (NBS) programme which can have a significant impact on long term clinical outcome. The need to repeat a sample because the initial sample is of insufficient quality for testing in an important contributor. These 'avoidable repeats' are monitored at maternity unit level as a key performance indicator. To check that NBS samples have a result in the recommended timeframes all maternity units in England use an IT system called the newborn blood spot failsafe solution (NBSFS). In North West England half of all delays in initial blood spot samples are for babies in a hospital setting. Babies that need to be admitted are more likely to have a screening positive condition and are also those that are the most likely to have a delay in initial sampling.

Objectives

- Comparison of avoidable repeat rates for babies in hospital settings compared to those in the community at the time of sampling

- Make recommendations for quality improvement in the timeliness of NBS screening completion for inpatient babies.

Methods Review of newborn blood spot failsafe solution data to see how it can be used to provide assurance of the completion of screening for babies in hospital at the time of sampling

Identification and analysis of laboratory data from one North West laboratory to calculate an avoidable repeat rate for babies that are in hospital at the time of sampling.

Feedback from quality assurance visits on processes to prompt timely initial blood spot screening and any repeat samples for babies on neonatal units and other inpatient settings.

Results NBSFS is incomplete for recording of inpatient status. The field is not mandated and the use of the term NICU does not allow for babies in other settings e.g. children's 
hospitals. Alternative methods of identification such as Day 0 samples were also found to be unreliable.

Analysing laboratory data for over 12,000 first samples received found an overall avoidable repeat rate of $2.1 \%$ (acceptable threshold $\leq 2.0 \%$, achievable threshold $\leq 1 \%$ ). An avoidable repeat rate of $5.2 \%$ was found for inpatient babies compared to $1.7 \%$ for community babies.

Badgernet is a neonatal IT system that issues screening reminder prompts in neonatal units to trigger completion of screening in neonatal units, these can be dismissed when screening is not complete. This system is not used in standalone children's hospitals.

Conclusions Laboratory data can provide maternity units with information to be able to identify differential avoidable repeat rates for babies in hospital settings. This allows the targeting of quality improvement work to reduce avoidable repeats.

NBSFS is currently not able to provide differential information about inpatient babies. This would be improved by renaming the 'NICU' field as 'inpatient' and mandating completion.

Access to NBSFS for neonatal units and other inpatient children's settings would support completion of timely screening. In the interim, local feedback mechanisms to update maternity units on screening status are needed.

\section{British Association of Perinatal Medicine and Neonatal Society}

\section{NEURALLY ADJUSTED VENTILATORY ASSIST (NAVA) IN VERY PREMATURELY BORN INFANTS WITH EVOLVING/ ESTABLISHED BPD}

${ }^{1}$ Sandeep Shetty, 'Katie Evans, 'Anay Kulkarni, 'Donovan Duffy, ${ }^{2}$ Anne Greenough. ${ }^{1}$ St George's Hospital NHS Foundation Trust; ${ }^{2}$ Asthma UK Centre for Allergic Mechanisms in Asthma, King's College London

\subsection{6/archdischild-2021-rcpch.290}

Background NAVA/NIV-NAVA (Neurally adjusted ventilatory assist/non-invasive NAVA) utilises the electrical activity of the diaphragm to trigger the ventilator. A modified nasogastric feeding tube with a series of electrodes allows monitoring of the diaphragmatic electromyogram (Edi). ${ }^{1}$ The waveform of the Edi is used to trigger and control ventilator support. NAVA/NIV-NAVA allows the infant to initiate support of inspiration and termination of inspiration, potentially allowing efficient ventilation at lower pressures. Furthermore, using the respiratory drive of the infant to control ventilation may help avoid hypocarbia and hypercarbia. ${ }^{2}$ Use of NAVA for infants requiring mechanical ventilation is in its infancy. ${ }^{3}$

Objectives Our aim was to determine whether NAVA/NIVNAVA has advantages over conventional modes of invasive and non-invasive ventilation in evolving/established (Bronchopulmonary dysplasia) BPD.

Methods A retrospective study was undertaken. Each infant on NAVA/NIV-NAVA was matched with two other infants (controls) supported by conventional invasive and NIV. Matching was by gestational age, birth weight, sex, antenatal steroid exposure and if inborn/or transferred ex utero. Infants were identified from a standardised electronic neonatal database (Badgernet). Data were obtained from the electronic documentation recording system. NAVA/NIV-
NAVA was delivered by the SERVO-n ${ }^{\circledR}$ Maquet Getinge ventilator and conventional ventilation (predominantly flow sensor triggered) by the Stephanie STEPHAN ventilator and non-triggered non-invasive modes were BiPAP, CPAP (flow driver) and HHFNC. Outcomes were extubation failure, duration of invasive and non-invasive ventilation, total length of hospital stay (LOS), BPD (oxygen requirement at 36 weeks corrected age) and home oxygen rates. Outcome included data from the local hospital after discharge from St George's Hospital (SGH). The study period was between June 2019 and November 2020. The infants were compared to the historical cohort born between June 2016 and January 2019. This project was registered with SGH Audit department.

Results Eighteen 'NAVA' infants were compared with 36 controls. Infants on NAVA/NIV NAVA had lower extubation failure rates (median $0(0-2)$ versus $1(0-6) \mathrm{p}=0.002)$, shorter durations of invasive ventilation (median 30.5 (1-90) days versus $40.5(11-199)$ days $p=0.046)$ and total duration of invasive and non-invasive ventilation up to the point of discharge from the local hospital (median 80 (57-140) days versus 103.5 (60-246) days $\mathrm{p}=0.026$ ). In addition, the total length of stay in hospital was lower in the NAVA/NIV-NAVA group (111.5 (78-183) days versus 140 days (82-266) days $\mathrm{p}=0.019)$. There were no differences in the BPD $(17 / 18$ $(94 \%)$ versus $32 / 36(89 \%) \mathrm{p}=0.511)$ or home oxygen rates $14 / 18(78 \%)$ versus 23/36 (64\%) $\mathrm{p}=0.305)$ between infants on NAVA/NIV NAVA group and infants in the control group. Conclusions These results suggest that a combination of NAVA/NIV-NAVA compared to conventional invasive and noninvasive modes may be advantageous for preterm infants with evolving/established BPD.

\section{REFERENCES}

1. Stein $H$, Firestone $K$. Application of neurally adjusted ventilatory assist in neonates. Semin Fetal Neonatal Med 2014;19(1):60-9.

2. Beck J. Patient-ventilator interaction during neurally adjusted ventilatory assist in low birth weight infants. Pediatr Res 2009;65(6):663-8.

3. Rossor TE, Hunt KA, Shetty S, Greenough A. Neurally adjusted ventilatory assist compared to other forms of triggered ventilation for neonatal respiratory support. Cochrane Database Syst Rev 2017:CD012251.

\section{International Child Health Group}

\section{ADDRESSING BARRIERS TO EARLY INTERVENTION IN CHILDREN WITH DEVELOPMENTAL IMPAIRMENT IN LUCKNOW, INDIA}

${ }^{1}$ Rahul Bharat, ${ }^{2}$ Uzaina Qazi, ${ }^{3}$ Sanjay Niranjan, ${ }^{3}$ TR Yadav. ${ }^{1}$ Association of Child Brain Research/Royal Cornwall Hospital NHS Trust; ${ }^{2}$ Association of Child Brain Research; ${ }^{3}$ Geniuslane Child development centre

\subsection{6/archdischild-2021-rcpch.291}

Background Child developmental impairment problems offer a challenge and a valuable opportunity. Unlike any other medical problem, the human brain continues to develop for the first six-year and respond to its environment. The parents are the main stakeholders for children and training parents could provide a valuable low-cost early intervention. The parents are often in denial about a developmental impairment. Some parents panic or feel anxious, angry, and hopeless. There seems to a lack of guidance on the best practice to address parental feelings. 